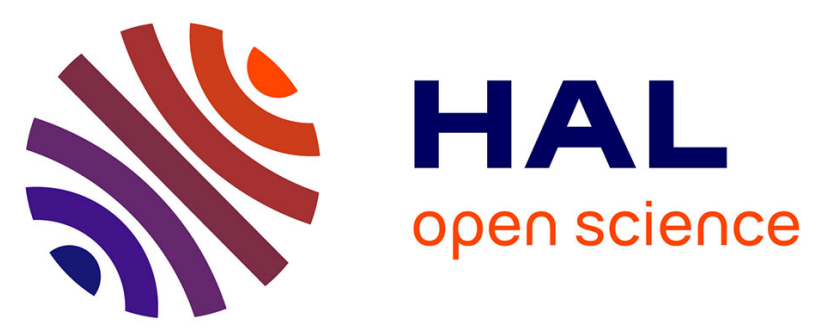

\title{
Modular Conjugation of a Potent Anti-HER2 Immunotoxin Using Coassociating Peptides
}

Audrey Stoessel, Nadja Groysbeck, Lucile Guyot, Lina Barret, Yves Nominé,

Leonel Nguekeu Zebaze, Ambre Bender, Laetitia Voilquin, Thomas Lutz, Nikita Pallaoro, et al.

\section{To cite this version:}

Audrey Stoessel, Nadja Groysbeck, Lucile Guyot, Lina Barret, Yves Nominé, et al.. Modular Conjugation of a Potent Anti-HER2 Immunotoxin Using Coassociating Peptides. Bioconjugate Chemistry, 2020, 31 (10), pp.2421-2430. 10.1021/acs.bioconjchem.0c00482 . hal-03005245

\section{HAL Id: hal-03005245 \\ https://hal.science/hal-03005245}

Submitted on 13 Nov 2020

HAL is a multi-disciplinary open access archive for the deposit and dissemination of scientific research documents, whether they are published or not. The documents may come from teaching and research institutions in France or abroad, or from public or private research centers.
L'archive ouverte pluridisciplinaire HAL, est destinée au dépôt et à la diffusion de documents scientifiques de niveau recherche, publiés ou non, émanant des établissements d'enseignement et de recherche français ou étrangers, des laboratoires publics ou privés. 


\section{Modular conjugation of a potent Anti-HER2 immunotoxin using co-associating peptides}

Audrey Stoessel ${ }^{1}$, Nadja Groysbeck ${ }^{1}$, Lucile Guyot ${ }^{2,3}$, Lina Barret ${ }^{2}$, Yves Nominé ${ }^{4}$, Leonel Nguekeu-Zebaze ${ }^{1}$, Ambre Bender ${ }^{1}$, Laetitia Voilquin ${ }^{4}$, Thomas Lutz ${ }^{1}$, Nikita Pallaoro ${ }^{1}$, Marie Blocat ${ }^{1}$, Celia Deville ${ }^{4}$, Murielle Masson ${ }^{1}$, Guy Zuber ${ }^{1}$, Bruno Chatton $^{1}$, and Mariel Donzeau ${ }^{1 *}$.

1 Université de Strasbourg, UMR7242 Biotechnologie et Signalisation Cellulaire, Ecole Supérieure de Biotechnologie Strasbourg, F-67412 Illkirch, France.

2 IMPReSs Facility, Biotechnology and Cell Signaling, CNRS - University of Strasbourg, Illkirch, F-67412 Illkirch, France.

3 NovAliX, Bioparc, F-67405 Illkirch, France

4 Institut de Génétique et de Biologie Moléculaire et Cellulaire, Illkirch, F-67400 Illkirch, France.

*mariel.donzeau@unistra.fr

\section{Abstract:}

Immunotoxins are emerging candidates for cancer therapeutics. These biomolecules consist of a cell targeting protein combined to a polypeptide toxin. Associations of both entities can be achieved either chemically by covalent bonds or genetically creating fusion proteins. However, chemical agents can affect activity and/or stability of the conjugate proteins and additional purification steps are often required to isolate the final conjugate from unwanted by-products. As for fusion proteins, they often suffer from low solubility and yield.

In this report, we describe a straightforward conjugation process to generate an immunotoxin using co-associating peptides (named K3 and E3), originating from the tetramerization domain of p53. To that end, a nanobody targeting the human epidermal growth factor receptor 2 (nano-HER2) and a protein toxin fragment from Pseudomonas aeruginosa Exotoxin A (TOX) were genetically fused to the E3 and K3 
peptides. Entities were produced separately in E. coli in soluble forms and at high yields. The nano-HER2 fused to the E3 or K3 helixes (nano-HER2-E3 and nanoHER2-K3) and the co-assembled immunotoxins (nano-HER2-K3E3-TOX and nanoHER2-E3K3-TOX) presented binding specificity on HER2 overexpressing cells with relative binding constants in the low nanomolar to picomolar range. Both toxin modules (E3-TOX and K3-TOX) and the combined immunotoxins exhibited similar cytotoxicity levels compared to the toxin alone (TOX). Finally, nano-HER2-K3E3-TOX and nano-HER2-E3K3-TOX evaluated on various breast cancer cells were highly potent and specific to kill HER2-overexpressing breast cancer cells with $\mathrm{IC}_{50}$ values in the picomolar range. Altogether, we demonstrate that this non-covalent conjugation method using two co-assembling peptides can be easily implemented for modular engineering of immunotoxins targeting different types of cancers.

Keywords: immunotoxin, co-assembly, peptide, HER2, cytotoxicity.

\section{INTRODUCTION}

The human epidermal growth factor receptor 2 (HER2) is a cell surface receptor overexpressed in about $15-20 \%$ of breast cancers. Amplification of HER2 is associated with tumor invasion and metastasis. Introduction of an anti-HER2 therapy using the humanized monoclonal antibody trastuzumab targeting specifically the extracellular domain of HER2 has largely improved patient care. ${ }^{1}$ However, intrinsically or acquired resistance has restricted the success of the trastuzumab. ${ }^{2,3}$ Since two decades, new anti-HER2 therapies have been under investigation. One emerging therapeutic is immunotoxins, which combine the specific targeting of antibodies with the high cytotoxic properties of bacterial or plant protein toxins. ${ }^{4-6}$ Upon specific binding to extracellular receptors and receptor-mediated endocytosis, immunotoxins get internalization into target cells, where the toxin domain unfolds its effect. These therapeutic biomolecules have demonstrated excellent anti-cancer properties at very low concentrations due to the enzymatic activity of the toxin fragment, which lowers the amount of molecules needed to kill cancer cells. ${ }^{7,8}$ One of the most widely used toxin fragments originates from Pseudomonas aeruginosa exotoxin A. It contains separate activities: a domain II for intracellular trafficking and a 
catalytic domain (domain III) inactivating the eukaryotic translation elongation factor 2 (eEF-2) by ADP-ribosylation. ${ }^{9}$

Immunotoxins can be produced as chimeric proteins. For instance, an affibody, an antibody mimetic molecule, directed against HER2 genetically fused to a modified exotoxin A fragment PE38 showed elevated cytotoxicity towards HER2 overexpressing cells. ${ }^{10,11}$ However, the weakness of such an approach is the expression level and the solubility issue of the recombinant immunotoxins. To overcome these problems, both the targeting and toxin moieties can be produced separately and are then chemically conjugated. ${ }^{12,13}$ For example, the scFv (single chain variable fragment) of trastuzumab and a minimal exotoxin A fragment (PE24) that were expressed independently and conjugated via a disulfide bond-containing linker, had strong cytotoxic effects on HER2 breast cancer cells. ${ }^{13}$ But the drawback of such an approach is the use of reducing agents, leading to improper protein folding, and therefore lowering solubility and/or activities of the conjugate proteins. Moreover, additional purification steps are required to isolate the desired conjugate from by-products present in the conjugation mixture. These additional purification steps often result in low yields and make the chemical conjugation a time-consuming and cost ineffective process.

In this study, we report a novel straightforward conjugation procedure based on co-assembly of a single chain antibody (nanobody or $\mathrm{VHH}$ ) targeting HER2 receptor and a truncated variant of the exotoxin A from Pseudomonas aeruginosa via two peptides (named E3 and K3), originating from the tetramerization domain of p53 (residues 325-355). ${ }^{14}$ These peptidic domains consist of a $\beta$-strand followed by a $\alpha$ helix. The $\alpha$-helical charged interface involving lysine and acid glutamic residues (E343, E346 and K351) modulates tetramer stability through salt bridges. Thus charge-reversal mutations at positions 343,346 and 351 was previously shown to favor heterotetramerization. ${ }^{15}$ While E3 (variant E343, E346 and E351) associates solely into dimers through $\beta$-strand interactions, K3 (variant K343, K346 and K351) forms tetramers with low stability. However, when mixed together in equimolar amounts, these peptides associate exclusively into dimer of primary dimers. This non-covalent interaction between E3 and K3 peptides was demonstrated to be strong and specific enough to allow heterotetramerization of macromolecules inside living cells for the engineering of bifunctional bio-molecules. ${ }^{16}$ Using this technology to coassemble a nanobody anti-HER2 with a truncated optimized fragment of the exotoxin 
A from Pseudomonas aeruginosa, we generate extremely stable heterotetrameric immunotoxins having a specific cytotoxic activity on HER2 positive breast cancer cells.

\section{RESULTS}

\section{Design of the immunotoxin}

We designed novel immunotoxins able to co-assemble via E3 and K3 peptides (Figure $1 \mathrm{~A}$ and $\mathrm{B}$ ). ${ }^{16}$ We choose a nanobody targeting the extracellular domain of HER2 (henceforth referred to as nano-HER2). ${ }^{17,18}$ The nano-HER2 sequence was genetically fused to the $\mathrm{N}$-terminal part of $\mathrm{K} 3$ sequence (Figure $1 \mathrm{~A}){ }^{16}$ The selected toxin module derives the Pseudomonas exotoxin A variant PE24, ${ }^{19,20}$ where the intracellular trafficking domain has been replaced by a furin cleavage site (FCS) described elsewhere ${ }^{21}$, and the B-cell epitopes and the protease-sensitive regions have been removed. ${ }^{20}$ This toxin part (hereinafter referred to as TOX) was then genetically fused to the C-terminal part of the E3 peptide (Figure 1A). The design of the immunotoxin was also carried out to obtain the opposite configuration, namely nano-HER2-E3 combined with K3-TOX, given rise to nano-HER2-E3K3-TOX (Figure 1B).

A

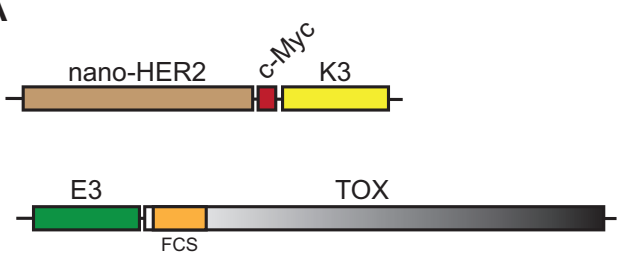

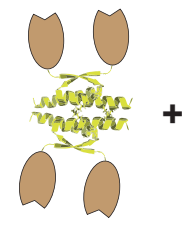

nano-HER2-K3

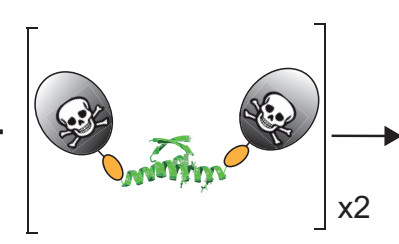

E3-TOX

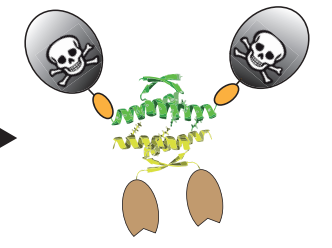

nano-HER2-K3E3-TOX

B

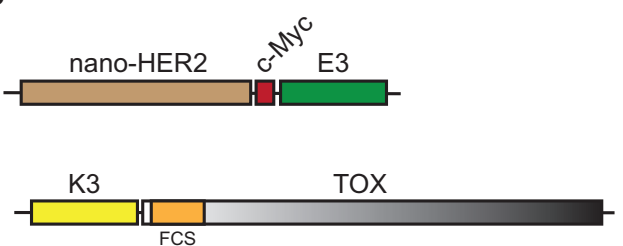

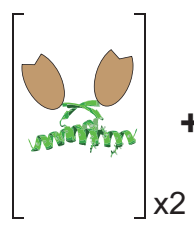

nano-HER2-E3

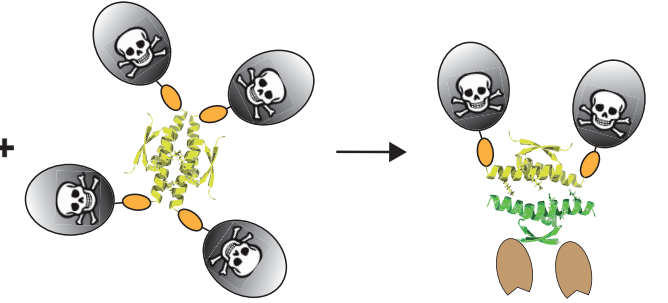

K3-TOX
nano-HER2-E3K3-TOX

Figure 1: Representations of heterotetrameric immunotoxins. Schematic representation of either nano-HER2-K3E3-TOX (A) or nano-HER2-E3K3-TOX (B) co-assembled immunotoxins with coding sequences of each module, as indicated. Theoretical oligomerization states of the corresponding proteins, and the resulting co-assembled immunotoxins are depicted. 


\section{Production, yield and oligomeric states of the recombinant proteins}

Recombinant $\mathrm{VHH}$ and TOX moieties were produced at high levels in $E$. coli (Figure S1A and S2A), and purified by immobilized metal affinity chromatography (IMAC) followed by a preparative size exclusion chromatography (SEC) (Figures S1B and $\mathrm{C}$, and Figure S2B and $\mathrm{C}$ ). Typical elution profiles revealed that the unfused nano-HER2 displayed a single peak with an apparent molecular weight of $21 \mathrm{kDa}$ corresponding to a theoretical molecular weight of a monomer, while nano-HER2-E3 and nano-HER2-K3 behaved like a 63 kDa dimer and a 105 kDa tetramer, respectively (Figure $2 \mathrm{~A}$ and Table $\mathrm{S1}$ ). TOX constructs gave the same results in terms of oligomeric states, namely a homodimer of $68 \mathrm{kDa}$ for E3-TOX and a homotetramer of $152 \mathrm{kDa}$ for K3-TOX, respectively (Figure 2A). In all cases, single peaks were observed indicating an oligomerization state of nearly $100 \%$. The final yields of the recombinant proteins after the two purification steps (Figures S1C and $\mathrm{S} 2 \mathrm{C}$ ) were around $100 \mathrm{mg} / \mathrm{L}$ for the nano-HER2-E3 and nano-HER2-K3, and in the range of 7 to $10 \mathrm{mg} / \mathrm{L}$ for the E3-TOX and K3-TOX (Table S2).

The secondary structure content of the recombinant proteins was next explored using far-UV circular dichroism (CD) spectroscopy, and the content of secondary structure elements was estimated using CDPro suite software. ${ }^{22}$ The farUV CD spectra of the tagged E3 and K3 constructs are highly similar (Figure 2B). The nano-HER2-E3 and the nano-HER2-K3 folded in $18 \% \pm 1$ and $16 \% \pm 1$ of $\alpha$-helix, compared to $5 \% \pm 2 \%$ for the parental nano-HER2, indicating a rough increase of 27 to 32 amino acids in $\alpha$-helix conformation per monomer (Figure 2B, and Table 1). These predictions are in agreement with previous results where adding E3 or K3 peptide to a con1 construct leads to an increased of almost 30 amino acid residues in $\alpha$-helix. ${ }^{16}$ Regarding the TOX proteins, the $\alpha$-helix portions increased in the similar way than for the nanobody constructs when compared to the parental TOX (Figure 2B and Table 1). Thus, secondary structures following addition of the $\mathrm{E} 3$ or $\mathrm{K} 3$ peptides stayed almost constant regardless of the constructs.

Finally, the thermal stability evaluation of the nano-HER2 recombinant proteins was performed using a fluorescence-based thermal shift assay (Figure S3). The melting profiles of the recombinant proteins were highly similar, indicating that fusion of the tagged constructs did not compromise the intrinsic stability of the VHH domain. 
A
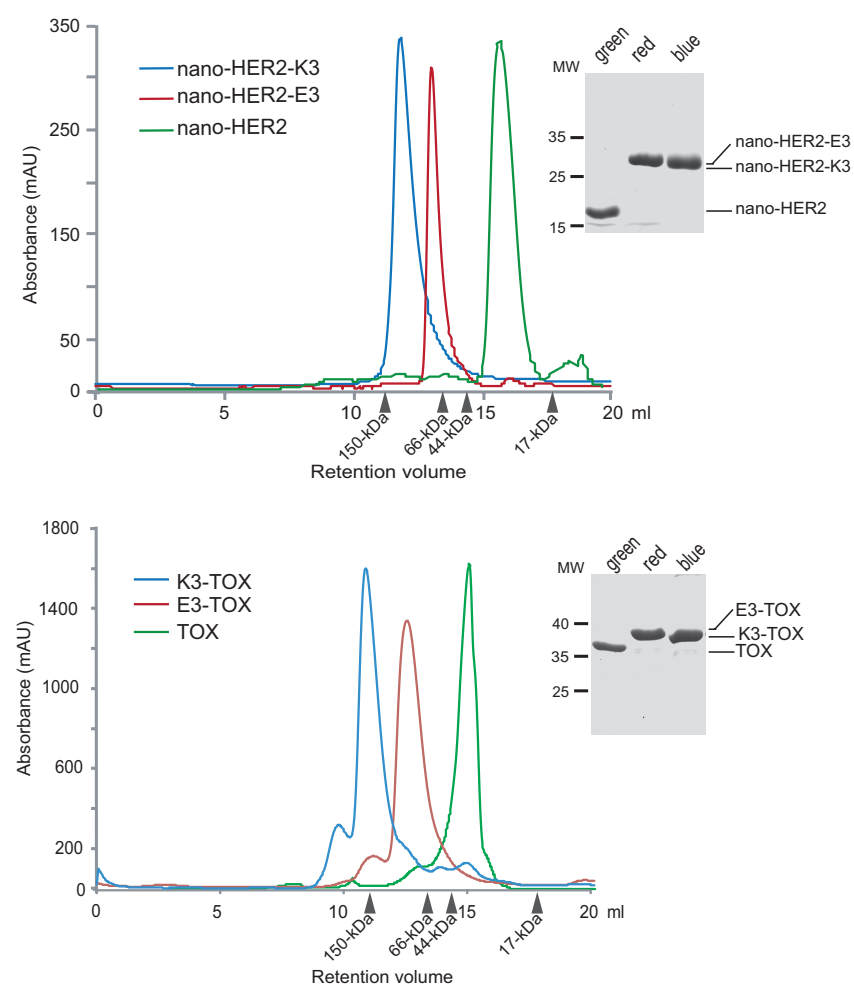

B
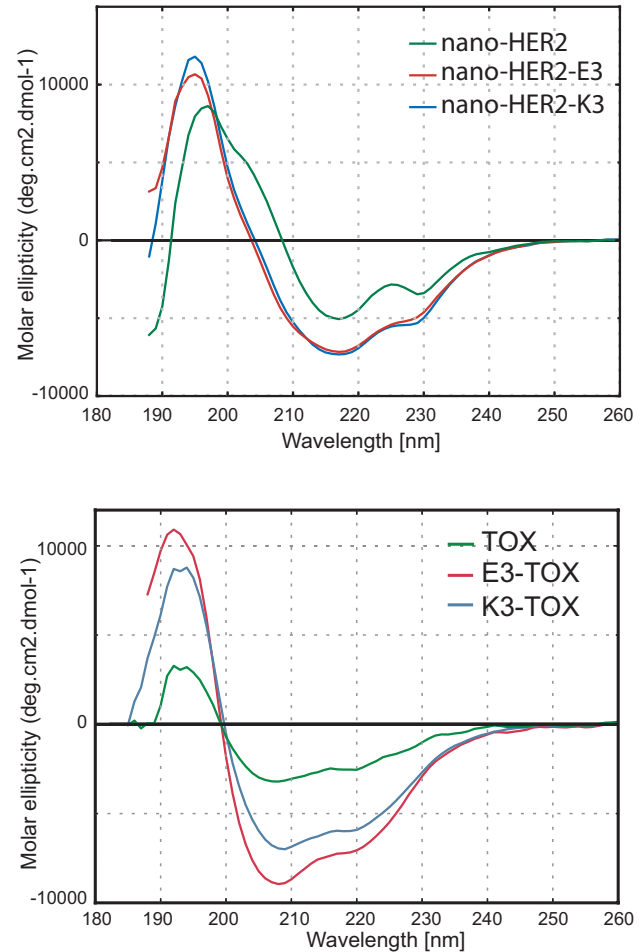

Figure 2. Physicochemical analysis of the $K 3$ and $E 3$ constructs. (A) Preparative gel filtration of purified nano-HER2 derivatives (above) and TOX constructs (below). The column was pre-calibrated with standard proteins as indicated (IgG, BSA, ovalbumin, and cytochrome C). A sample of each peak was analyzed by SDS-PAGE and by Coomassie blue staining. (B) Far-UV circular dichroism spectra of the indicated assemblies were recorded for samples at a monomeric concentration of $69 \mu \mathrm{M}$ in phosphate saline buffer and are presented as an average of ten successive scans. CD signal is expressed in mean residue ellipticity $\left(\mathrm{deg} \cdot \mathrm{cm}^{2} \cdot \mathrm{dmol}^{-1}\right.$ ). Data were collected in the $185-270 \mathrm{~nm}$ range at $20^{\circ} \mathrm{C}$.

Table 1: Secondary structure contents of the constructs: aa refers to the numbers of amino acids determined considering the percentage of each secondary structure content and assuming a single element.

\section{ALPHA}

BETA

OTHERS

\begin{tabular}{lcccccc}
\hline & $\%$ & aa & $\%$ & aa & $\%$ & aa \\
Nano-HER2 & $5 \pm 2$ & 7 & $41 \pm 8$ & 61 & $54 \pm 9$ & 79 \\
Nano-HER2-E3 & $19 \pm 1$ & 39 & $30 \pm 3$ & 64 & $52 \pm 2$ & 110 \\
Nano-HER2-K3 & $16 \pm 1$ & 34 & $33 \pm 3$ & 70 & $51 \pm 3$ & 109 \\
TOX & $7 \pm 2$ & 20 & $39 \pm 3$ & 114 & $54 \pm 4$ & 156 \\
E3-TOX & $20 \pm 2$ & 61 & $28 \pm 2$ & 88 & $53 \pm 3$ & 166 \\
K3-TOX & $17 \pm 1$ & 53 & $31 \pm 2$ & 98 & $52 \pm 2$ & 164
\end{tabular}




\section{In vitro formation of the heterotetramer complexes}

Following the two purification steps, we studied the efficiencies of coassociation between the nanobody and toxin modules in various conditions. Evaluation of modularity and efficiency of heteromerization were first analyzed in phosphate saline buffer at $\mathrm{pH} 7.4$ using size exclusion chromatography (SEC). When equimolar amounts of either nano-HER2-E3 and K3-TOX, or nano-HER2-K3 and E3TOX proteins were mixed together and analyzed by SEC, an apparent $125 \mathrm{kDa}$ complex was produced, which was compatible with the theoretical molecular weight of the heterotetramer (Figure $3 A$ and $B$, and Table S1). Thus, both elution profiles confirmed that the homotetramer dissociates to the benefit of a more stable heterotetrameric complex. As the extracellular micro-environment of a solid tumor is acidic, $^{23}$ the stability of the co-assembled immunotoxin nano-HER2-K3E3-TOX was assessed at low pH. Equimolar amount of nano-HER2-K3 and E3-TOX were mixed together and run on size exclusion chromatography in PBS pH 6. As shown in figure 3C, the nano-HER2-K3E3-TOX complex runs exactly at the same apparent molecular weight than the complex form at $\mathrm{pH}$ 7.4. Thus the nano-HER2-K3E3-TOX is stable at $\mathrm{pH} 6$.

The quality of the protein assemblies in regard to colloidal stability was monitored by Dynamic Light Scattering analysis (DLS)(Figure S4). All complexes, including the heterotetramers display apparent hydrodynamic diameters of size below $14 \mathrm{~nm}$ with a low polydispersity index (PDI) in a range of 0.20 , with the complete absence of unwanted protein-aggregates. 

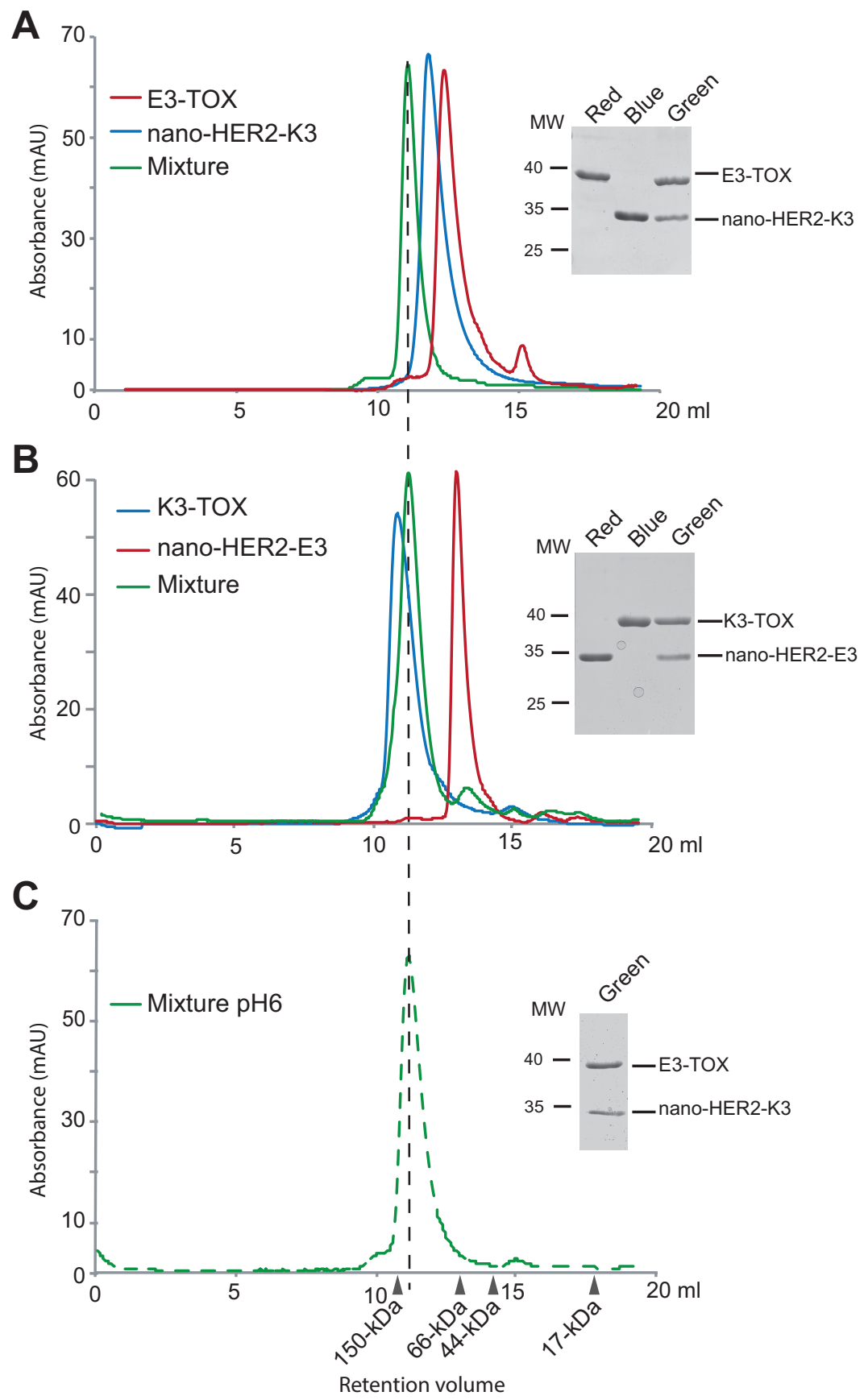

Figure 3. Co-association of the immunotoxins. Gel filtration of (A) purified nano-HER2-K3 (blue), E3TOX (red), and a mixture of equimolar amounts of nano-HER2-K3 and E3-TOX (green), (B) purified nano-HER2-E3 (red), K3-TOX (blue), and a mixture of equimolar amounts of nano-HER2-E3 and K3TOX (green), and analysis by SDS-PAGE and Coomassie blue staining of the peak fractions. (C) Gel filtration of co-assembled nano-HER2-K3/E3-TOX immunotoxin run at $\mathrm{pH} 6$ (dotted green line) and SDS-PAGE and Coomassie staining analysis of the peak fraction. 


\section{The nanobodies and the co-associated immunotoxins retain cell-binding properties.}

To assess whether the formation of heterodimers via E3 and K3 helixes does not affect the binding of the nano-HER2, binding efficiencies of nanobodies and coassembled immunotoxins were then measured on HER2-positive HCC1954 cells to determine relative binding constants. Thus, the recombinant proteins were randomly conjugated to NHS-Alexa488 dye via lysine residues and compared to the labeled parental nano-HER2 (Figure 4A and S5A). Following incubation with HCC1954 cells, fluorescence signals were measured using fluorescence-activated cell sorting (FACS). As shown in Figure 4B, monovalent nano-HER2 displayed an apparent binding constant $\left(\mathrm{EC}_{50}\right)$ of $21.8 \mathrm{nM}( \pm 4.8)$, somewhat lower than the value previously described elsewhere on HER2-positive SKBR3 cells. ${ }^{17}$ The same experiment performed with the homodimer nano-HER2-E3 and the homotetramer nano-HER2$\mathrm{K} 3$ exhibited relative binding efficiency values $1.0 \mathrm{nM}( \pm 0.1)$ and $0.2 \mathrm{nM}( \pm 0.1)$ indicating an increased binding efficiency of around 20- and 100-fold, respectively. These results pointed to an avidity effect of the dimer and tetramer nanobodies for improved binding to HER2 receptor. However, labeling either E3- or K3-TOX module prior assembly with the respective nanobody counterpart resulted in a significant drop in binding constants (Figure S5B). This result was likely due to a steric hindrance of the fluorophore preventing association between the toxin and the $\mathrm{VHH}$ modules as shown by analytical gel filtration (Figure S5C). Thus, labeling purified immunotoxin complexes resolved this issue (Figure S5). Indeed, Alexa488-labeled immunotoxin complexes displayed only a slight lower binding efficiency than their respective nanobody counterparts (Figure 4A and 4B). Nano-HER2-K3E3-TOX immunotoxin exhibited an $\mathrm{EC}_{50}$ of $2.5 \mathrm{nM} \pm 0.3$ and the nano-HER2-E3K3-TOX immunotoxin an $\mathrm{EC}_{50}$ of $3.7 \mathrm{nM} \pm 0.4$. No detectable binding was observed on HER2 low-expressing cells MDA-DB-231 or on HER2-silenced HCC1954 cells using smallinterfering oligonucleotides (Figure S6A and S6B).

Overall, these results demonstrate the binding efficiency and specificity of the co-associated immunotoxins on HER2 cell surface receptor. 
A

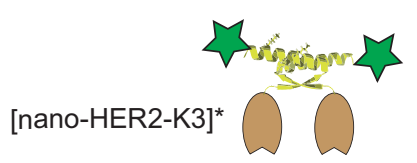

[nano-HER2-K3E3-TOX] *
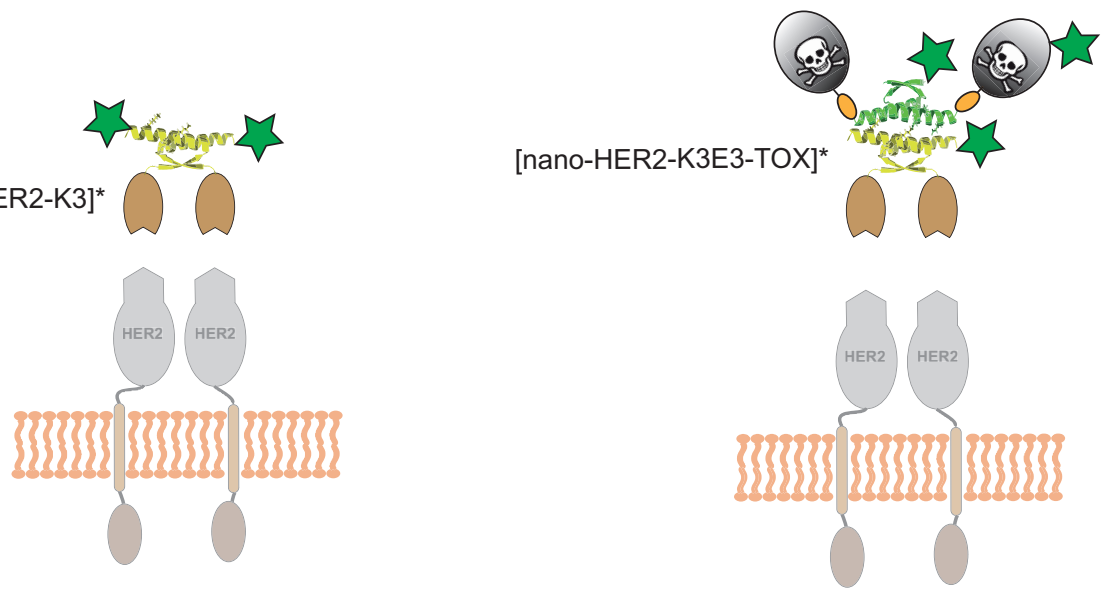

B

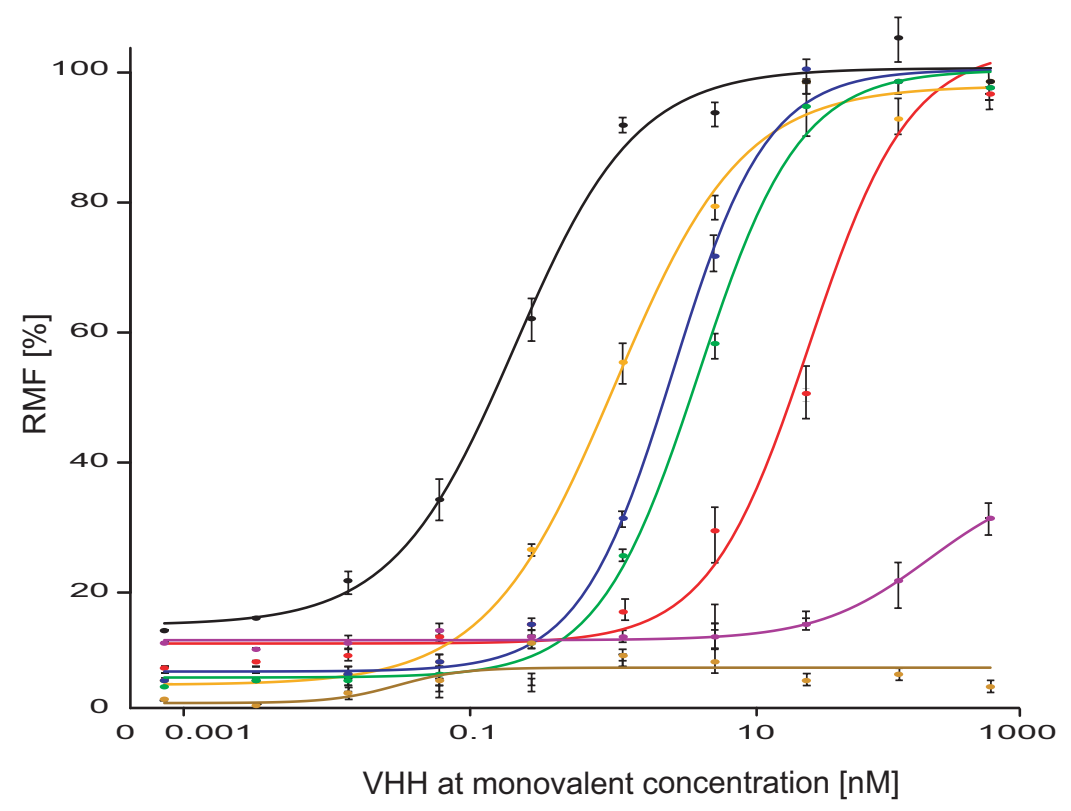

- [nano-HER2-K3] ${ }^{*}$

- [nano-HER2-E3]

- [nano-HER2-E3K3-TOX] ${ }^{*}$

- [nano-HER2-K3E3-TOX] ${ }^{*}$

- [nano-HER2]

- $[\mathrm{E} 3-\mathrm{TOX}]^{*}$

-PBS

Figure 4. Apparent binding constants of the constructs on antigen-overexpressing cells. (A) Cartoon representing the binding of the labeled constructs [*] on HER2 cell surface receptor. (B) HCC1954 cells were titrated by increasing amounts of Alexa488-labeled recombinant proteins, as indicated [*]. Following incubation, the fluorescence was measured by fluorescence-activated cell sorting (FACS). The relative mean of fluorescence (RFM) was plotted against nanobody concentration (at monovalent concentration), and Kd values were determined using sigmoidal fitting with $R$ software. ( $n \geq 6)$ and SD.

\section{Co-assembly of the toxin with the nanobody via E3 or K3 peptides does not impair its cytotoxicity}

Because oligomerization may harm the structural and functional integrity of the toxin, we evaluated the enzymatic activities of the toxins and the immunotoxins using an in vitro coupled transcription/translation assay using rabbit reticulocyte lysate and a luciferase reporter gene (Figure 5A). Increasing concentrations of either TOX, E3- 
TOX, K3-TOX, or the co-assembled immunotoxins were added to the lysate in the presence of NAD+, the essential cofactor for the ADP-ribosylation of the eEF-2 elongation factor. ${ }^{24}$ Translation efficiencies were monitored by measuring luciferase activities (Figure 5A). Determination of the inhibition dose-response curve yielded a half-inhibition $\left(\mathrm{IC}_{50}\right)$ at $235 \mathrm{pM}( \pm 45)$ for the TOX comparable to the E3-TOX at 164 pM ( \pm 30 ), and both co-assembled immunotoxins, HER2-E3K3-TOX and HER2K3E3-TOX with IC50 of 160 pM ( \pm 15$)$ and 260 pM $( \pm 20)$, respectively. The homotetramer K3-TOX showed a slightly reduce inhibition activity with an IC50 of $610 \mathrm{pM}( \pm 50)$. Thus, addition of the helix and co-assembly of the nanobody with the toxin via E3 or K3 peptides did not compromise the enzymatic activity of the toxin.

The cytotoxic activity of all constructs were then also assessed by electrotransferring the recombinant proteins and complexes into various cell lines. ${ }^{25}$ Both TOX and E3-TOX moieties yielded more than $90 \%$ of cell death after $72 \mathrm{~h}$ (Figure 5B). As a control, the transduced nano-HER2-E3 did not have any cytotoxic effects.

A

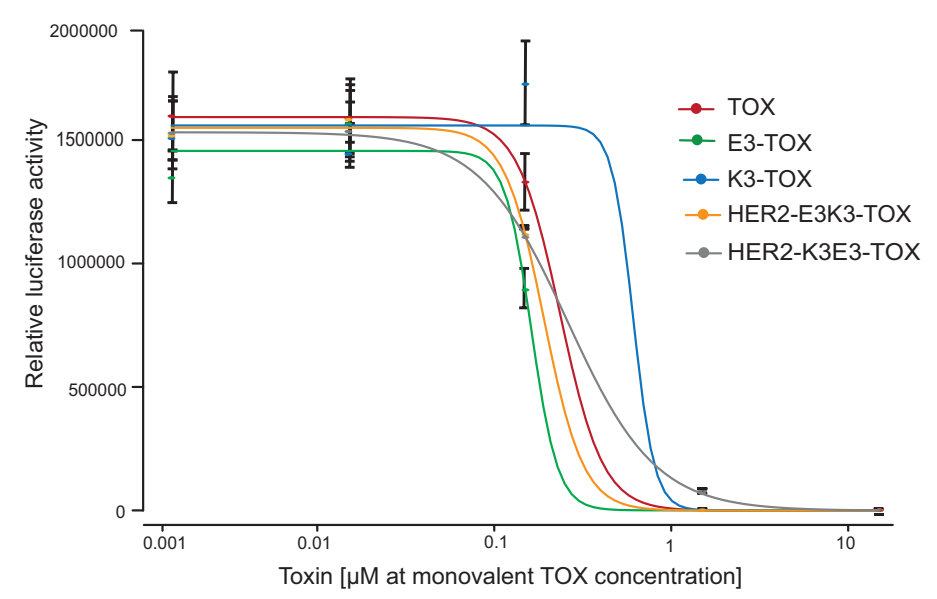

B

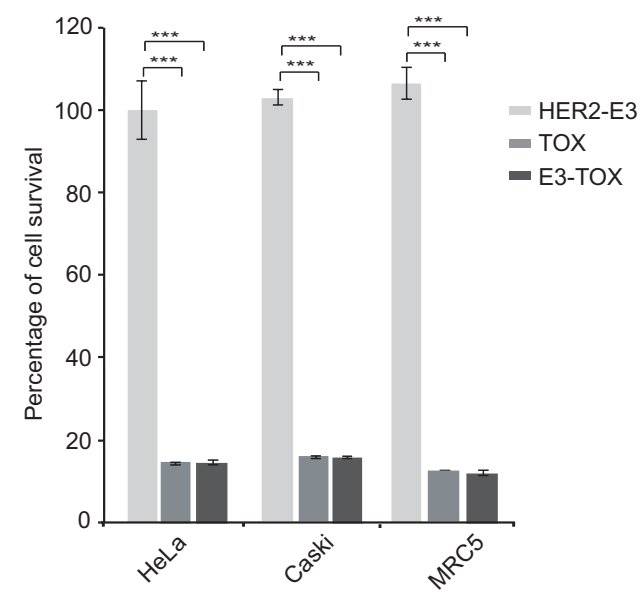

Figure 5: Cytotoxicity of the TOX modules. (A) Toxins and Immunotoxins inhibit protein translation. Recombinant proteins were tested in an in vitro transcription/translation assay using rabbit reticulocyte lysate and the luciferase activity as reporter. This assay was performed in triplicate. Sigmoidal curve fitting was performed using R software. (B) TOX and E3-TOX purified proteins were electro-transferred into various cell lines. After $72 \mathrm{~h}$, cell viability was estimated as a percentage relative to cells transduced with PBS buffer. Analysis corresponds to at least an average of three independent experiments. Error bars represent standard deviations. Student's t-test was performed: ${ }^{*} \mathrm{P}<0.05,{ }^{* *} \mathrm{P}<0.01,{ }^{* * *} \mathrm{P}<0.001$. 


\section{Specific cytotoxicity of the immunotoxins on HER2-positive breast cancer cells}

Given the high binding affinity of the nano-HER2 for HER2 positive cancer cells and the high cytotoxicity of the TOX modules, we sought to determine the cytotoxicity of the co-assembled immunotoxins.

Thus, the cytotoxic effect and the specificity of the co-assembled immunotoxin, nano-HER2-K3E3-TOX, were evaluated on different breast cancer cell lines being either HER2-positive or HER2-negative. Three HER2-positive (HCC1954, BT474 and SBKR3), two HER2-negative (MCF7 and MDA-MB-231), and a non-human myoblastic cell line (H9C2(2-1) rat) were tested. ${ }^{26}$ The cells were incubated for $72 \mathrm{~h}$ with increasing concentrations of either the immunotoxin nano-HER2-K3E3-TOX or the nano-HER2-K3 or the E3-TOX complexes (Figure 6A and 6B). The nano-HER2K3E3-TOX revealed high cytotoxicity at picomolar levels on the HER2 overexpressing cell lines, compared to the toxin and to the $\mathrm{VHH}$ alone. The $\mathrm{IC}_{50}$ values for BT474, HCC1954 and SKBR3 cells were $30 \mathrm{pM}( \pm 4), 39 \mathrm{pM}( \pm 15)$, and 160 pM $( \pm 25)$, respectively $(n \geq 10)$ (Figures $6 A$, and Table 2$)$. The toxin alone, E3TOX, had lower cytotoxic effects, in the range of 5 to $10 \mathrm{nM}$ for HER2-positive cells and for MCF7 cells (Table 2). Thus the nano-HER2-K3E3-TOX immunotoxin was 185, 595 and 65 fold more potent on HCC1954, BT474 and SKBR3 cells, respectively, compared to the free toxin (E3-TOX) (Figure 6A and Table 2). No significant differences between the free toxin and the immunotoxin were noted in HER2-negative cells, supporting the notion that HER2 overexpression increases the uptake of the immunotoxin (Figure 6B and Table 2). In fact, MCF7, HER2-negative cell line, were affected to the same extent by the free toxin and by the immunotoxin with an $\mathrm{IC}_{50}$ from 8.02 to $6.20 \mathrm{nM}( \pm 0.15)$. Of note, the viability of MDA-MB-231 as well as $\mathrm{H} 9 \mathrm{C} 2(2-1)$ cell lines, was not affected by the immunotoxin $\left(\mathrm{IC}_{50} \geq 300 \mathrm{nM}\right)$ (Figure 6B, and Table 2). Similarly, the opposite configuration, namely the nanoHER2-E3K3-TOX displayed the same cytotoxicity as the nano-HER2-K3E3-TOX on HER2-positive cell lines and no effects on MDA-MB-231 (Figure S7 and data not shown).

The proteolytic stability of the constructs was also assessed by incubating both immunotoxins in $80 \%$ of serum (FBS) at $37{ }^{\circ} \mathrm{C}$ during $1,5,12$ and $24 \mathrm{~h}$. Immunotoxin cytotoxicity was then tested on HER2-positive HCC1954 cells. NanoHER2-K3E3-TOX retained its full cytotoxic activity even after $24 \mathrm{~h}$ incubation in 
serum at $37^{\circ} \mathrm{C}$ with an IC50 of $27.0 \mathrm{pM}( \pm 6.3)$ (Figure $\left.6 \mathrm{C}\right)$.

Altogether, these results indicate that the cytotoxicity of both immunotoxins is dependent on the specific association between the nano-HER2 and the toxin via the E3 and K3 helixes, and correlates with the presence of the HER2 receptor on the cell's surface. In addition, they show that the immunotoxin complexes are very stable at $\mathrm{pH} 6.00$ as well as in serum.

A

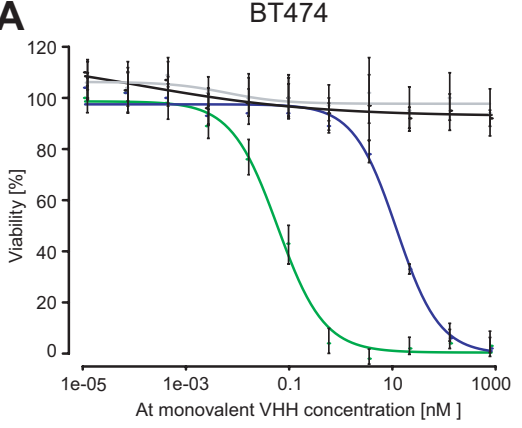

B

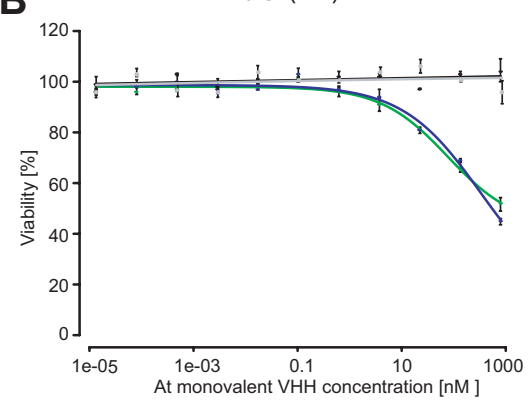

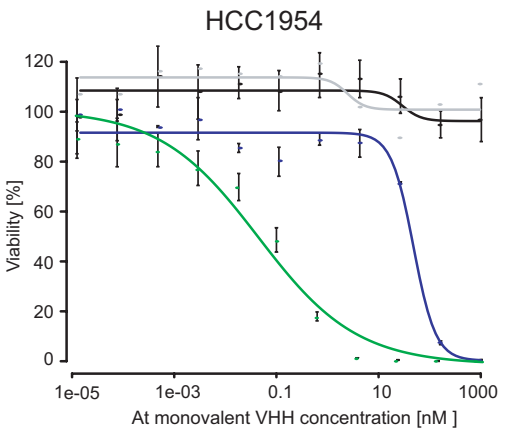
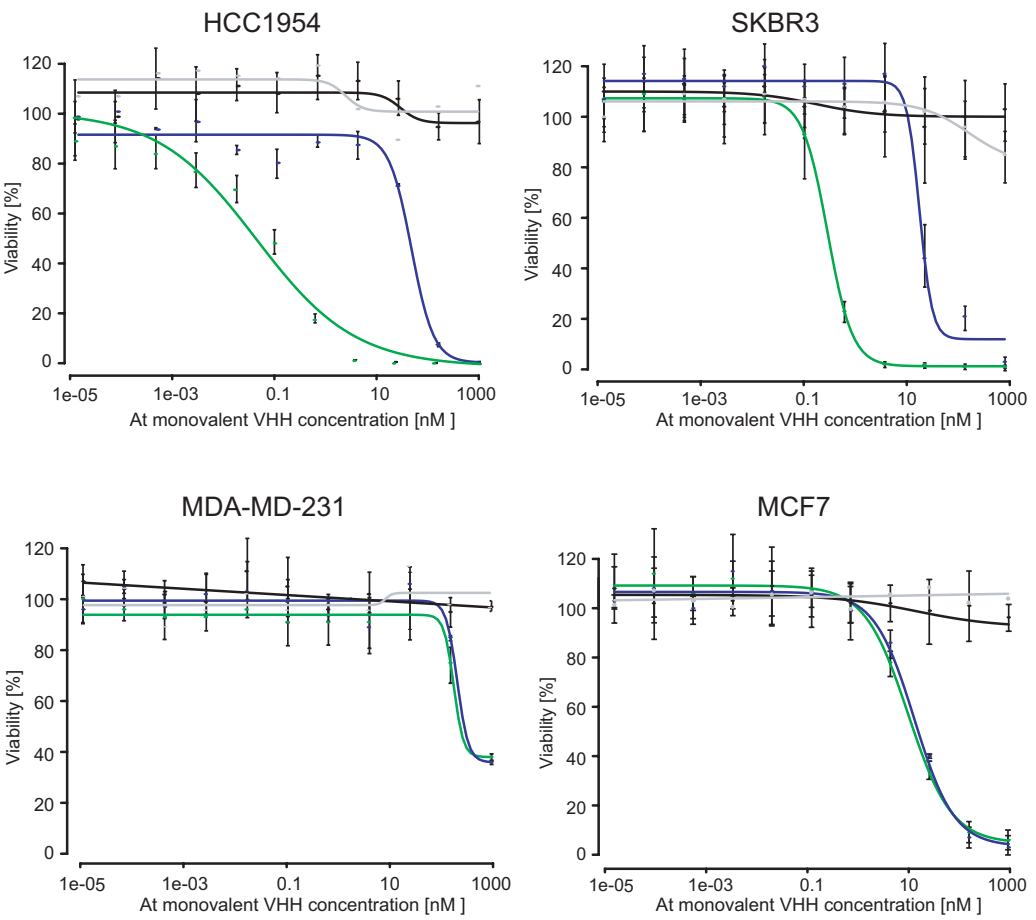

- nano-HER2-K3E3-TOX

- nano-HER2-K3

- E3-TOX

- Control

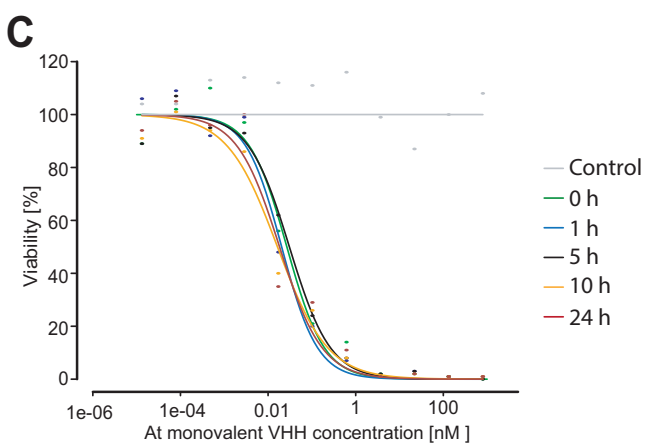

Figure 6. The immunotoxin nano-HER2-K3E3-TOX shows high cytotoxicity and specificity for HER2 overexpressing cells. (A) HER2-positive and (B) HER2-negative cells were treated for $72 \mathrm{~h}$ with the nano-HER2-K3E3-TOX, nano-HER2-K3, and E3-TOX alone $(n \geq 10)$. (C) HER2-positive HCC1954 cells were treated for $72 \mathrm{~h}$ with the nano-HER2-K3E3-TOX previously incubated in $80 \%$ serum at $37^{\circ} \mathrm{C}$ for 
various times, as indicated. HER2 The relative cell viability to untreated cells was plotted against antibody concentrations, and $\mathrm{IC}_{50}$ values were determined using sigmoidal fitting with $\mathrm{R}$ software.

Table 2. Cytotoxicity of the immunotoxin on target and control cells.

\begin{tabular}{lccccc}
\hline Bio-molecule & $\begin{array}{c}\text { BT474 } \\
\text { IC }_{50}(\mathbf{p M})\end{array}$ & $\begin{array}{c}\text { HCC1954 } \\
\text { IC }_{50}(\mathbf{p M})\end{array}$ & $\begin{array}{c}\text { SKBR3 } \\
\text { IC }_{50}(\mathbf{p M})\end{array}$ & $\begin{array}{c}\text { MDA-MB-231 } \\
\text { IC }_{50}(\mathbf{p M})\end{array}$ & $\begin{array}{c}\text { MCF7 } \\
\text { IC }_{50}(\mathbf{p M})\end{array}$ \\
\hline $\begin{array}{l}\text { Nano-HER2- } \\
\text { K3E3-TOX }\end{array}$ & $30.0 \pm 4.0$ & $39.0 \pm 15$ & $160 \pm 25$ & $>300000$ & $6250 \pm 150$ \\
E3-TOX & $5550 \pm 80$ & $16710 \pm 150$ & $10380 \pm 230$ & $>300000$ & $8020 \pm 150$ \\
$\begin{array}{l}\text { Nano-HER2- } \\
\text { K3 }\end{array}$ & $\mathrm{NA}$ & $\mathrm{NA}$ & $\mathrm{NA}$ & $\mathrm{NA}$ & $\mathrm{NA}$ \\
Ratio ER & 185 & 595 & 65 & & n.d. \\
\hline
\end{tabular}

\section{DISCUSION}

In this study, we developed a straightforward co-assembly technique to produce an immunotoxin combining a nanobody directed against HER2 and a toxin via two peptides E3 and $\mathrm{K} 3 .{ }^{16}$ The non-covalent and highly specific pairing of the E3 and $\mathrm{K} 3$ peptides yielded to the generation of heterotetrameric immunotoxins, composed of two $\mathrm{VHH}$ and two toxin molecules. We clearly demonstrate that the engineered immunotoxins retain their specific binding and toxic properties, hence being specifically toxic for HER2-overexpressing breast cancer cells.

Because production of immunotoxins is impeded in eukaryotic cells due to their high toxicity, most engineering applications for their production relies on the design of chimeras produced in E. coli. ${ }^{10,11,20}$ However, fusion proteins often suffer from low solubility and low yield. ${ }^{10,27}$ For example, an affibody anti-HER2 fused to a PE38 fragment from the exotoxin A of Pseudomonas aeruginosa was not soluble and was purified from the inclusion bodies ${ }^{10}$ while a chimeric recombinant immunotoxin composed of a $\mathrm{VHH}$ directed against the vascular endothelial growth factor receptor 2 and the PE38 fragment was produced at a final yield of $9.2 \mathrm{mg} / \mathrm{L} .{ }^{27}$ Another strategy is to generate both moieties independently, which in addition to improved yield and solubility, makes it possible to produce the antibody domain in eukaryotic cells. Different protein conjugation strategies have been explored. For instance, a HER2-scFv fragment has been chemically conjugated to PE24 via N-succinimidyl-3(2-pyridyldithio)propionate (SPDP). ${ }^{13}$ The HER2-(scFv)-PE24 end product was then 
purified by size exclusion chromatography. However, the highest yield of the obtained conjugation was only $58 \%$ and required additional purification steps. An elegant protein trans-splicing (TPS) technology was also reported for the conjugation of either the Trastuzumab-targeted HER2 or two VHHs targeting different epitopes of HER1 (EGFR) to the PE24 fragment of the Exotoxin A from Pseudomonas. ${ }^{12}$ Coupling efficiencies in the range of $50-70 \%$ were achieved and it was not possible to distinguish molecules with a toxin/antibody ratio of 2 from those with partial coupling. Finally, an additional step was also required to remove the unconjugated $\mathrm{mAb}$.

The use of co-assembling peptides offers several significant advantages. Both moieties can be produced separately, overcoming solubility and yield issues reported for chimeric immunotoxins. Indeed, the $\mathrm{VHH}$ was produced at a yield of $100 \mathrm{mg} / \mathrm{L}$ and the toxin module showed a high solubility yield. In addition, neither reduction nor chemical conjugation is required, thereby avoiding subsequent purification steps, making it a very simple and convenient technology. Following assembly of the nanoHER2 and the toxin via E3 and K3, the heterotetramer immunotoxin complexes were able to efficiently and specifically kill HER2-expressing breast cancer cells. In addition, the bivalence format of the nanobody can probably trigger an efficient cellular uptake through receptor clustering. ${ }^{28,29}$ Upon endocytosis, the toxin part can be cleaved from the immunotoxin by the furin protease at the FCS in the early endosome and the enzymatic domain can reach the cytosol, where it finally inhibits translation. ${ }^{8}$ The cytotoxicity of the immunotoxins was highly specific to HER2 overexpressing cells with an efficacy of more than 65-fold compared to the toxin alone, or to HER2 low expressing or HER2 negative cells.

The present study describes a very straightforward process for generating an immunotoxin without chemical conjugation and additional purification steps to remove unreacted compounds and side products from the desired conjugation product. The association between E3 and K3 helices ensures a highly homogenous end product. In addition, this fully modular technology enables the replacement of the nano-HER2 moiety with any other target-specific binding domain to target other types of cancer cells. Finally, the possibility to produce both entities separately overcomes the solubility and yield issues of a full recombinant immunotoxin and offers the possibility 
to express the target moiety in an eukaryotic organism, to achieve post-translational modifications if needed, for proper folding or activity.

\section{Experimental procedures}

\section{Cell Lines}

HCC1954, MDA-MB-231, BT474 and H9C2 (2-1) cell lines were maintained as monolayers in RPMI without Hepes, supplemented with $10 \%$ fetal calf serum (FCS) and Gentamycin. MCF7, Caski, HeLa, MRC5 and SBKR cell lines were maintained as monolayers in Dulbecco's modified Eagle's medium (DMEM) (1 g/L Glucose) supplemented with $10 \%$ fetal calf serum (FCS) and Gentamycin. For BT474 and MCF7, the medium was supplemented with $10 \mu \mathrm{g} / \mathrm{ml}$ Insulin.

\section{Recombinant plasmid constructions}

Nano-HER2 DNA sequences described elsewhere ${ }^{18}$ were amplified by PCR using HER2-For GATATACCATGGAAGTTCAACTGG and HER2-Rev ATGTGCACTAGTTGCGGCCGCAGAGCTAACCGTCACTTGGGTACC primers. HER2 PCR fragment was digested with Ncol-Spel and ligated into the pETOMP40M1-E3 or -K3 digested with Ncol-Spel to replace the P40M1 sequences. ${ }^{16} \mathrm{An}$ optimized sequence encoding for the TOX used in this study was synthetized by Integrated DNA Technologies (IDT) and amplified by PCR using specific primers: For-TOX TCTACTAGTGCAATGGGGTCTGGTGGCTGT and Rev-TOX: GAGCTTAAGAATAATGTTAAGTAGAAAG. The resulting PCR fragment digested with Spel-EcoRI with has been then cloned into Spel-EcoRI of the pETOM-E3 and pETOM-K3. ${ }^{16}$ The E3 and K3 sequences were amplified by PCR using Nhel-K3-For GCTCGCTAGCGGTAACAACACCAGCTCCTCTC and HindIII-K3-Rev GCTCAAGCTTTTAACCCCCTGGCTCCTTCCCAGCC oligonucleotides and cloned into a pET6His-eGFP vector.

\section{Expression and purification of the recombinant fusion proteins}

Briefly, E3 and K3 fusion proteins were overexpressed in E. coli BL21 (DE3) pLysS with $0.5 \mathrm{mM}$ isopropyl thiogalactoside (IPTG). After $24 \mathrm{~h}$ at $20^{\circ} \mathrm{C}$, the cells were harvested, re-suspended $20 \mathrm{mM}$ Phosphate buffer $\mathrm{pH} 8,250 \mathrm{mM} \mathrm{NaCl}$ and 10 $\mathrm{mM}$ imidazole. Following lysis in a cell disruptor (Constant Systems Ltd), cell debris were removed by ultra-centrifugation, and the supernatant was applied to IMAC 
chromatography charged with cobalt (GE Healthcare Saclay, France). IMAC purified proteins were subsequently loaded on HiLoad 16/60 Superdex 200 prep grade column or on Superdex 200 Increased 10/300 (GE Healthcare, Bio-sciences AB, Sweden) operating at a flow rate of $0.5 \mathrm{ml} / \mathrm{min}$. Fractions were separated on SDSPAGE gels and analyzed by Coomassie blue. The heterotetramers were prepared by incubating together E3 and K3 targeted recombinant proteins at 1:1 molar ratio 5 min at RT followed in some experiments by a second purification on Superdex 200 Increased 10/300 GL size exclusion chromatography. Complex formation between labeled recombinant proteins was control by analytical chromatography on Superdex 200 Increased 5/150 GL.

\section{Protein labeling}

Recombinant proteins were labeled with either NHS-Ester Alexa488 dye using DyLight TM Microscale Antibody Labeling Kit following the protocol as described by the manufacturer (Thermo-Scientific, USA). Labeled proteins are indicated as $\left.{ }^{\star}\right]$ in text and figures.

\section{In vitro protein transcription/translation inhibition assay.}

Inhibition of protein translation was performed using rabbit reticulocyte lysate (RRL) (Promega) and by measurement of luciferase activity. The assay was performed as suggested by the manufacturer (Promega) in a total volume of $15 \mu \mathrm{l}$. Briefly, RRL was mixed with T7 expression plasmid encoding for the firefly luciferase and incubated for $90 \mathrm{~min}$ at $30{ }^{\circ} \mathrm{C}$ with or without increasing concentration of recombinant TOX. Then $5 \mu \mathrm{l}$ of each reaction were transferred to a black 96-well plate and assayed for luciferase activity, as previously described. ${ }^{30} \mathrm{~A}$ control without any protein added served as positive control and was set as relative protein translation of $100 \%$. At least three independent experiments were carried out.

\section{Circular Dichroism}

$\mathrm{CD}$ experiments were performed at $20.0 \pm 0.1{ }^{\circ} \mathrm{C}$ on a Jasco J-815 spectropolarimeter with $0.1 \mathrm{~mm}$ path-length quartz-Suprasil cells (JASCO Inc., Easton, MD USA). Acquisition parameters as continuous scan rate, response time, and bandwidth were $50 \mathrm{~nm} / \mathrm{min}, 1.0 \mathrm{~s}$, and $1 \mathrm{~nm}$, respectively. The absorbance of the 
buffer and the sample was kept as low as possible to ensure good signal-to-noise ratio. All spectra are systematically corrected by subtracting the solvent spectrum obtained under identical conditions.

\section{Thermal Shift Assay}

TSA experiments were carried out on real-time PCR systems (StepOnePlus; Applied Biosystems, Darmstadt, Germany). Purified nano-HER2, nano-HER2-E3 and nano-HER2-K3 were mixed with diluted Sypro Orange dye (final 2500-fold dilution from stock solution; Invitrogen, Carlsbad, CA, USA) in Phosphate Buffered Saline, $\mathrm{pH} 7.4$ to a final volume of $20 \mu \mathrm{l}$ and a final protein concentration of $5 \mu \mathrm{M}$. The samples were submitted to a denaturation kinetic from 25 to $95{ }^{\circ} \mathrm{C}$ at a rate of $1^{\circ} \mathrm{C} / \mathrm{min}$. The fluorescence of Sypro Orange dye was recorded in real time (excitation with a blue LED source and emission filtered through a ROX emission filter). The fluorescence profiles were fitted to a Boltzmann sigmoid equation to determine the melting temperature. Each experiment was repeated four times.

\section{Dynamic Light Scattering analysis (DLS)}

DLS was performed at $25^{\circ} \mathrm{C}$ using different protein concentration on a Malvern Zetasizer devise (Malvernpanalytical). Results analysis was performed using Zetasizer software.

\section{Transient siRNA transfections}

Transient siRNA transfections were performed using Lipofectamine RNAiMAX (Invitrogen, P/N 56532) according to the manufacturer's instructions. STARD3targeting siRNAs and HER2-targeting siRNAs were SMARTpool ON-TARGETplus obtained from Dharmacon. For controls, siRNAs ON-TARGETplus non-targeting pool from Dharmacon were used. siRNAs were used at $10 \mathrm{nM}$ final concentration and cells were transfected $24 \mathrm{~h}-72 \mathrm{~h}$ prior to experiments.

\section{Fluorescence-Activated Cell Sorting (FACS)}

Trypsinized cells were incubated with a mixture of equimolar ratio of recombinant proteins as indicated, for $30 \mathrm{~min}$ at $4{ }^{\circ} \mathrm{C}$ in PBS, 0.5\% BSA, $2 \mathrm{mM}$ EDTA. Cells were washed twice in PBS, 0.5\% BSA, 2 mM EDTA and analyzed with 
BD Accuri ${ }^{\mathrm{TM}}$ C6 Plus flow cytometer (BD Bioscience). The relative mean fluorescence intensities were normalized and plotted against the concentration of the nano-HER2 at monomer concentration. The data shown here are single point measurements.

\section{Cytotoxicity Assay}

5000 cells were seeded per well in a 96-well plate and incubated for $24 \mathrm{~h}$. Cells were then treated with increased concentrations of toxin, $\mathrm{VHH}$, or immunotoxins. After 72 h, a crystal violet assay was conducted. ${ }^{31}$ The absorbance was measured in a Tecan reader $(595 \mathrm{~nm})$. $I_{50}$ values were calculated by fitting a sigmoidal model with R software. Wells with cells treated with PBS were set at $100 \%$ of cell viability.

\section{Supporting Information Available}

DNA and proteins sequences, figures showing additional representations of the immunotoxin, purification steps and SEC analysis of recombinants proteins, thermal stability profiles, and cells viability assays with recombinant immunotoxin.

\section{Acknowledgements}

This work was supported by funds and/or fellowships from the Centre National de la Recherche Scientifique, the University of Strasbourg, the French Ministry of Research and the Ligue Régionale contre le Cancer (CCIR-GE). The CD device was funded with the help of the Association pour la Recherche sur le Cancer (ARC n.8008). Lucile GUYOT is supported by a grant from the French ANRT agency (CIFRE N²018/1643). We thank C.L. Tomasetto and J.C. Amé for very helpful advises, and R. Wagner for the anti-HER2 nanobody clone.

\section{References}

(1) Romond, E. H., Perez, E. A., Bryant, J., Suman, V. J., Geyer, C. E., Davidson, N. E., Tan-Chiu, E., Martino, S., Paik, S., Kaufman, P. A., Swain, S. M., Pisansky, T. M., Fehrenbacher, L., Kutteh, L. A., Vogel, V. G., Visscher, D. W., Yothers, G., Jenkins, R. B., Brown, A. M., Dakhil, S. R., Mamounas, E. P., Lingle, W. L., Klein, P. M., Ingle, J. N., and Wolmark, N. (2005) Trastuzumab plus adjuvant chemotherapy for operable HER2-positive breast cancer. N Engl J 
Med 353, 1673-1684.

(2) Heyde, von der, S., Wagner, S., Czerny, A., Nietert, M., Ludewig, F., Salinas-Riester, G., Arlt, D., and Beissbarth, T. (2015) mRNA Profiling Reveals Determinants of Trastuzumab Efficiency in HER2-Positive Breast Cancer. PLoS ONE (Tan, M., Ed.) 10, e0117818.

(3) Chakrabarty, A., Bhola, N. E., Sutton, C., Ghosh, R., Kuba, M. G., Dave, B., Chang, J. C., and Arteaga, C. L. (2013) Trastuzumab-Resistant Cells Rely on a HER2-PI3K-FoxO-Survivin Axis and Are Sensitive to PI3K Inhibitors. Cancer Res 73, 1190-1200.

(4) Simon, N., and FitzGerald, D. (2016) Immunotoxin Therapies for the Treatment of Epidermal Growth Factor Receptor-Dependent Cancers. Toxins 8, 137.

(5) Kreitman, R. J. (2006) Immunotoxins for targeted cancer therapy. AAPS J 8, E532-51.

(6) Wayne, A. S., Fitzgerald, D. J., Kreitman, R. J., and Pastan, I. (2014) Immunotoxins for leukemia. Blood 123, 2470-2477.

(7) Tsuchikama, K., and An, Z. (2016) Antibody-drug conjugates: recent advances in conjugation and linker chemistries 1-14.

(8) Wolf, P., and Elsässer-Beile, U. (2009) Pseudomonas exotoxin A: From virulence factor to anti-cancer agent. International Journal of Medical Microbiology 299, 161-176.

(9) Michalska, M., and Wolf, P. (2015) Pseudomonas Exotoxin A: optimized by evolution for effective killing. Front. Microbiol. 6, 958.

(10) Amiri Tehranizadeh, Z., Sankian, M., Fazly Bazzaz, B. S., Chamani, J., Mehri, S., Baratian, A., and Saberi, M. R. (2019) The immunotoxin activity of exotoxin A is sensitive to domain modifications. Int. J. Biol. Macromol. 134, 1120-1131.

(11) Guo, R., Cao, L., Guo, W., Liu, H., Xu, H., Fang, Q., and Hong, Z. (2016) HER2-targeted immunotoxins with low nonspecific toxicity and immunogenicity. Biochem Biophys Res Commun 475, 93-99.

(12) Pirzer, T., Becher, K.-S., Rieker, M., Meckel, T., Mootz, H. D., and Kolmar, H. (2018) Generation of Potent Anti-HER1/2 Immunotoxins by Protein Ligation Using Split Inteins. ACS Chemical Biology 13, 2058-2066.

(13) Lee, S., Park, S., Nguyen, M. T., Lee, E., Kim, J., Baek, S., Kim, C. J., Jang, Y. J., and Choe, H. (2019) A chemical conjugate between HER2-targeting antibody fragment and Pseudomonas exotoxin A fragment demonstrates cytotoxic effects on HER2-expressing breast cancer cells. BMB Reports 52, 496-501.

(14) Brokx, R. D., Bolewska-Pedyczak, E., and Gariépy, J. (2003) A stable human p53 heterotetramer based on constructive charge interactions within the tetramerization domain. J Biol Chem 278, 2327-2332.

(15) Brokx, R. D., Bolewska-Pedyczak, E., and Gariépy, J. (2003) A Stable Human p53 Heterotetramer Based on Constructive Charge Interactions within the Tetramerization Domain. J Biol Chem 278, 2327-2332.

(16) Vigneron, M., Dietsch, F., Bianchetti, L., Dejaegere, A., Nominé, Y., Cordonnier, A., Zuber, G., Chatton, B., and Donzeau, M. (2019) Self-Associating Peptides for Modular Bifunctional Conjugation of Tetramer Macromolecules in Living Cells. Bioconjugate Chem. 30, 1734-1744.

(17) Kijanka, M., Warnders, F.-J., Khattabi, El, M., Lub-de Hooge, M., van Dam, G. M., Ntziachristos, V., de Vries, L., Oliveira, S., and van Bergen en Henegouwen, P. M. P. (2013) Rapid optical imaging of human breast tumour xenografts using anti-HER2 VHHs site-directly conjugated to IRDye $800 \mathrm{CW}$ for image-guided surgery. Eur J Nucl Med Mol Imaging 40, 1718-1729.

(18) Hartmann, L., Botzanowski, T., Galibert, M., Jullian, M., Chabrol, E., Zeder Lutz, G., 
Kugler, V., Stojko, J., Strub, J.-M., Ferry, G., Frankiewicz, L., Puget, K., Wagner, R., Cianferani, S., and Boutin, J. A. (2019) VHH characterization. Comparison of recombinant with chemically synthesized anti-HER2 VHH. Protein Science 5, 1122.

(19) Hollevoet, K., Mason-Osann, E., Liu, X. F., Imhof-Jung, S., Niederfellner, G., and Pastan, I. (2014) In Vitro and In Vivo Activity of the Low-Immunogenic Antimesothelin Immunotoxin RG7787 in Pancreatic Cancer. Molecular Cancer Therapeutics 13, 2040-2049.

(20) Bauss, F., Lechmann, M., Krippendorff, B.-F., Staack, R., Herting, F., Festag, M., ImhofJung, S., Hesse, F., Pompiati, M., Kollmorgen, G., da Silva Mateus Seidl, R., Bossenmaier, B., Lau, W., Schantz, C., Stracke, J. O., Brinkmann, U., Onda, M., Pastan, I., Bosslet, K., and Niederfellner, G. (2016) Characterization of a re-engineered, mesothelin-targeted Pseudomonasexotoxin fusion protein for lung cancer therapy. Molecular Oncology 10, 13171329.

(21) Kaplan, G., Lee, F., Onda, M., Kolyvas, E., Bhardwaj, G., Baker, D., and Pastan, I. (2016) Protection of the Furin Cleavage Site in Low-Toxicity Immunotoxins Based on Pseudomonas Exotoxin A. Toxins 8, 217.

(22) Sreerama, N., and Woody, R. W. (2000) Estimation of Protein Secondary Structure from Circular Dichroism Spectra: Comparison of CONTIN, SELCON, and CDSSTR Methods with an Expanded Reference Set. Anal Biochem 287, 252-260.

(23) Wike-Hooley, J. L., Haveman, J., and Reinhold, H. S. (1984) The relevance of tumour pH to the treatment of malignant disease. Radiother Oncol 2, 343-366.

(24) Pastrana, D. V., and Fitzgerald, D. J. (2006) A nonradioactive, cell-free method for measuring protein synthesis inhibition by Pseudomonas exotoxin. Anal Biochem 353, 266271.

(25) Freund, G., Sibler, A.-P., Desplancq, D., Oulad-Abdelghani, M., Vigneron, M., Gannon, J., Van Regenmortel, M. H., and Weiss, E. (2013) Targeting endogenous nuclear antigens by electrotransfer of monoclonal antibodies in living cells. mabs 5, 518-522.

(26) Dai, X., Cheng, H., Bai, Z., and Li, J. Breast Cancer Cell Line Classification and Its Relevance with Breast Tumor Subtyping. J. Cancer 8, 3131-3141.

(27) Behdani, M., Zeinali, S., Karimipour, M., Khanahmad, H., Schoonooghe, S., Aslemarz, A., Seyed, N., Moazami-Godarzi, R., Baniahmad, F., Habibi-Anbouhi, M., Hassanzadeh-

Ghassabeh, G., and Muyldermans, S. (2013) Development of VEGFR2-specific Nanobody Pseudomonas exotoxin A conjugated to provide efficient inhibition of tumor cell growth. New Biotechnology 30, 205-209.

(28) Moody, P. R., Sayers, E. J., Magnusson, J. P., Alexander, C., Borri, P., Watson, P., and Jones, A. T. (2016) Trigger Internalization and Lysosomal Targeting of Therapeutic Receptor:Ligand Complexes. Molecular Therapy 23, 1888-1898.

(29) Wang, Q., Villeneuve, G., and Wang, Z. (2005) Control of epidermal growth factor receptor endocytosis by receptor dimerization, rather than receptor kinase activation. $E M B O$ Rep 6, 942-948.

(30) Diring, J., Camuzeaux, B., Donzeau, M., Vigneron, M., Rosa-Calatrava, M., Kedinger, C., and Chatton, B. (2011) A cytoplasmic negative regulator isoform of ATF7 impairs ATF7 and ATF2 phosphorylation and transcriptional activity. PLOS ONE 6, e23351.

(31) Feoktistova, M., Geserick, P., and Leverkus, M. (2016) Crystal Violet Assay for Determining Viability of Cultured Cells. Cold Spring Harb Protoc 2016, pdb.prot087379. 